\title{
On Sufficiency and Invariance
}

D. BAST", University of Ohicago and

Indian Statistical Institute

\section{SLMMARY}

Let $\left(\mathscr{C}, \mathcal{A}, \mathscr{D}^{\prime}\right)$ be a given statistical model and let $\mathscr{\mathcal { L }}$ be the class of all one-to-one, bimeascuabie maps $g$ of $(\mathscr{C}, \mathscr{A})$ onto itself such that $g$ is measure-preserving for each $P \varepsilon x^{\prime}$, i.e. $P_{q^{-1}}=P$ for all $P$. Let us suppose that there exists a least (minimal) sufficient sub-field $\mathcal{L}$. Them, for each $L_{\mathcal{E}} \mathcal{L}$, it is true that $g^{-1} /$

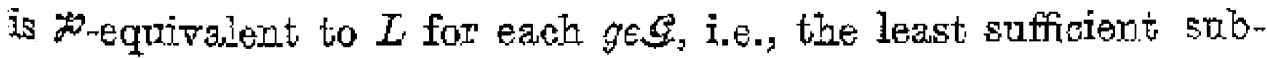
aid is almost $G$-invariant. It is demonstrated that, in many familier statistical models, the least sufficients sub-field and the sah-feid oî all almost $\mathcal{A}$-invariant sets aro indeed po-equivalent. The problem of data reduction in the presence of nuisance parameters has been discussed very briefly. It is shown that in rong situations the principle of invariance is strong enough to lead us to the standard reductions. For instance, given $n$ independent observations on a normal tariable with unknown mean

\footnotetext{
His resenrch was stpported by Research Grant Tro. INSF GP.3707 from

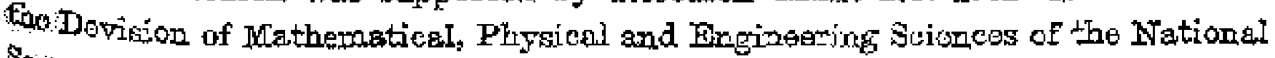

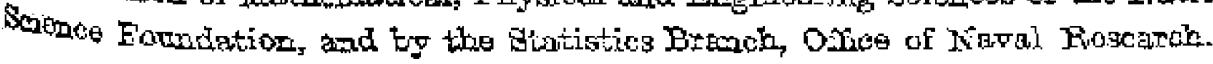


(the nuisance parameter) and unknown variance, it is shown how the principle of invariance alone can reduce the data to the sample variance.

\section{DEFINITIONS AND PRELIMINARIES}

(a) The basic probability model is denoted by $(\mathscr{C}, \mathcal{A}, \infty$ where $\mathscr{C}=\{x\}$ is the sample space, $\mathscr{A}=\{A\}$ the $\sigma$-field of events and $j o=\{P\}$ the family of probability measures.

(b) By set we mean a typical member of $\mathcal{A}$. By function (usually denoted by $f$ ) we mean a measurable mapping of $(\mathcal{A}, \mathscr{A}$ ) into the real line.

(c) A set $A$ is 70 -null if $P(A)=0$ for all $P \in \not D$. Two sets $A_{1}$ and $A_{2}$ are 70 -equivalent if their symmetric difference is to-null. Two functions $f_{1}$ and $f_{2}$ are $x_{\text {-equivalent if the set of }}$ points where they differ is 70 -null. The relation symbol $\sim$ stands for 70 -equivalence.

(d) By sub-field we mean a sub- $\sigma$-field of $\mathcal{A}$. A statistic is a measurable mapping of $(\mathscr{C}, \mathscr{A})$ into any measurable space. We identify a statistic with the sub-field it induces (see pp. $36-39$ of [6]).

(e) By the $\not p$-completion $\overline{\mathscr{A}}_{0}$ of a sub-field $\mathscr{A}_{0}$ we mean the least sub-field that contains $\mathscr{A}_{0}$ and all $\mathcal{F}_{\text {-null sets. Observe that }}$ $\bar{A}_{0}$ may also be characterized as the class of all sets that are to-equivalent to some member of $\mathscr{A}_{0}$. Two sub-fields are op-equivalent if they have identical 70 -completions.

(f) By transformation (usually denoted by $g$ ) we mean a oneto-one, bimeasurable mapping $g$ of $(\mathscr{C}, \mathscr{A})$ onto itself such that the family

$$
\not D g^{-1}=\left\{P g^{-1} \mid P \in \not O S\right\}
$$

of induced probability measures is the same as the family to. A transformation $g$ is called model-preserving if

$$
P g^{-1} \equiv P \text {, for all } P \in \neq^{\circ} \text {. }
$$


observe that, if $g$ is any transformation, then so also is $g^{n}$ for each integral (positive or negative) $n$ and that the identity map is always model-preserving. Also observe that any transformation carries $j^{0}$-null sets into 70 -null sets.

(g) Given a transformation $g$, the sub-field $\mathcal{A}(g)$ of $g$-invariant sets is defined as

$$
\mathcal{A}(g)=\left\{A \mid g^{-1} A=A\right\} .
$$

The $f^{0}$-completion $\bar{A}(g)$ of $\mathscr{A}(g)$ is then the class of all essentially

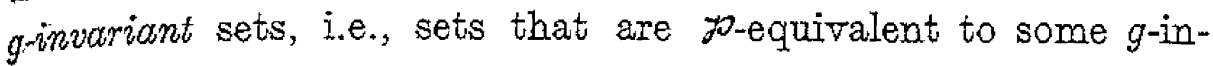
variant set.

(h) The set $A$ is almost $g$-invariant if $g^{-1} A \sim A$. It is easy to demonstrate that every almost $g$-invariant set is also essentially $g$-invariant and vice versa (see Lemma I for a sharper result). Thus, $\overline{\mathcal{A}}(g)$ is also the class of all almost $g$-invariant sets.

(i) Given a class $\mathcal{S}$ of transformations $g$, the three sub-fields of a) $\mathcal{S}$-invariant, $\beta$ ) essentially $\mathcal{S}$-invariant and $\gamma$ ) almost $\mathcal{G}$-invariant sets are defined as follows:

a) $\mathscr{A}(\mathcal{S})=\bigcap \mathcal{A}(g),(\mathcal{E}$-invariant)

B) $\overline{\mathscr{A}}(\mathcal{S})=\not 0$-completion of $\mathcal{A}(\mathcal{S})$, (essentially $\mathcal{G}$-invariant) and

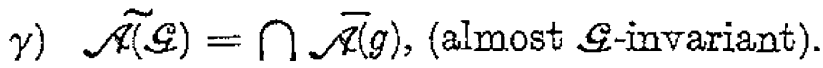

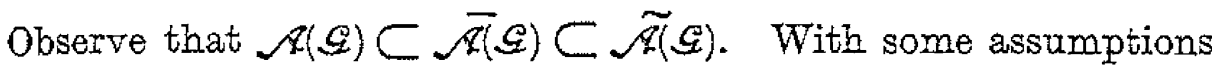
on $\mathcal{G}$, one can prove (see Theorem 4 on p. 225 in [6]) the equality of $\overline{\mathscr{A}(\mathcal{S})}$ and $\widetilde{\mathscr{A}}(\mathcal{S})$. That $\overline{\mathcal{A}(\mathcal{G})}$ can be a very small sub-field compared to $\widetilde{\mathcal{A}(\mathcal{G})}$ is shown in example 1 .

(j) A function $f$ is $\alpha$ ) $\mathcal{E}$-invariant, $\beta$ ) essentially $\mathcal{G}$-invariant, or $\gamma$ ) almost $\mathcal{S}$-invariant, according as

a) $f=f(g)$, for all $g \in \mathcal{S}$,

B) $f \sim$ some $\mathcal{S}$-invariant function, or

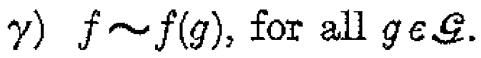


Observe that $f$ satistios the defnitions $\alpha$ ), $\beta$ ) or $\gamma$ ) above if and only if $f$ is measurable with respect to the corresponding subfield defined in (i).

(k) When the class $\mathcal{G}$ of transformations $g$ happens to be a group (with respect to the operation of composition of transformations), the sub-field,$R(S)$ of $\{$-invariant sets is easily recognized as follows. For oach $z \in \mathscr{Q}$, define the orbit $O_{x}$ as

$$
O_{\mathrm{c}}=\left\{x_{1}^{\prime} x^{\prime}=g x \text { for some } g \in \mathcal{G}\right\} \text {. }
$$

The orbits define a partition of $\mathscr{C}$, and the sub-field $\mathscr{R}(\mathcal{S})$ is the class of all (measurable) sets that are the tuions of orbits. The sub-ineld of essentially $S$-invariant sets is then the zo-completion of $\mathcal{A}(\mathcal{S})$. Our main concern, in this paper, is the sub-feld $\widetilde{A}(s)$ and this is not so easily understood in terms of the orbitsunless 9 has a suructare simple enough to ensure the equaity of $\overline{x(C)}$ and $\widetilde{A(S)}$ (see lemma 1 and example 1 ).

\section{A MATHEMATIGAL INTRODUGTYON}

Let $\left(\mathcal{C}, \mathcal{A}, X^{O}\right)$ be a given probability model and let $g$ be a fixed model-preserving transformation [definition 2(f)]. We remarl that the identity map is trivially model-preserving. In many instances of statistical interest there exist fairly wide classes of such transformations. However, it is not dificult to construct examples where no non-trivial transformation is model-preserring. See for instance examplo 5 .

For each bounded function $f$ [definition $2(0)$, define the associated sequence $\left\{f_{n}\right\}$ of (uniformly bounded) functions as follows:

$$
f_{n}(x)=\left[f(x)+f(g x)+\ldots+f\left(g^{n} x\right)\right] /(n+1), \quad n=1,2,3, \ldots
$$

Since $g$ is measure-preserving for each $P \in D_{0}$, the pointwise ergodic theorem toils us that the set $N_{f}$ where $\left\{f_{n}(x)\right\}$ fails to converge is $\sigma^{2}$-nill [definition $\left.2(c)\right]$.

If we dofine $f^{*}$ as

$$
f^{*}(x)= \begin{cases}\lim f_{x}(x) & \text { when } x \in N_{f} \\ 0 & \text { othorwise }\end{cases}
$$


then, it is easily seen that

i) $f^{*}$ is $\mathscr{A}(g)$-measurable [definition $2(g)$ ], i.e., it is $g$-invariant [definition 2(i)], and that

ii) for all $B \in \mathscr{A}(g)$ and $P \in X$

$$
\int_{B} f a P=\int_{B} f^{*} d P
$$

This is another way of saying that $f^{*}$ is the conditional expectation of $f$ given the sub-field $\mathscr{A}(g)$ of $g$-invariant sets. Since the definition of $f^{*}$ does not involve $P$, we have the following theorem (lemma 2 in [4]).

Theorem 1. If the transformation og proserves the modet $\left(\mathscr{N}, \mathcal{A}, x_{1}\right.$, then the sib-fiedd $\mathcal{A}(g)$ is sufficient.

[Remark: Note that in the proof of theorem I we have not osed the assumptions that $g$ is a one-to-one map and that it is bimeasurable. The proof remains valid for any measurable mapping of $(\mathscr{C}, \mathcal{A})$ into itself that is measure-preserving for each $P \in D D$. A similar remank will hold true for a number of other rosults to be stated later. Howerex, in a study of the statistical theory of invarianee (see [6]) it seems appropriate to restriot owr attention to one-to-one, bimeasurable maps of $(\mathfrak{C}, \mathfrak{A})$ onto $x^{2}$ self that prescrve the model either wholly or partially.]

Now, given a class $\mathcal{E}$ of model-preserving transformations $g$, what can we say about the sufficiency of the sub-field

$$
\mathscr{A}(\mathcal{S})=\cap \mathscr{A}(g)
$$

of $\mathcal{G}$-invariant [definition $2(\mathrm{i})$ ] sets ? The intersection of two sufficient sub-ficlds is not necessarily suffioient. However, it is kaown (se Theorem 4 and Corollary 2 of [3]) that the intersection of the -completions [definition 2(e)] of a countable muraber of sufficient sub-fields is safficient. Csing this result, We tiave the following theorem (theorem 2 in [4]).

Theorem 2. If the alass $S$ of model-preserving transjormations 2 is countable, then the sub-field

$$
\tilde{\mathcal{A}}(\mathcal{E})=\cap \overline{\mathcal{X}}(g)
$$


of aimost $\mathcal{G}$-invariant sets [definition $2(i)(\gamma)$ ] is sufficiont.

Given a comtable class $\mathcal{G}$ of transformations, consider the larger class $\mathcal{S}^{*}$ of transformations of the type $\alpha_{1} \alpha_{2} \ldots \alpha_{n 2}$, where oach $\alpha_{i}$ is such that either $\alpha_{i}$ or $\alpha_{i}^{-1}$ belongs to $\mathcal{S}$, and $n$ is an arbitrary positive integer. The following properties of $\mathcal{G}^{*}$ are easy to check:

a) $\mathcal{G}^{*}$ is a group the group operation being composition of trunsformations),

b) $\mathcal{G}^{*}$ is countable,

c) $\mathscr{A}\left(G^{*}\right)=\mathscr{A}(G)$ and $\widetilde{A}\left(\mathscr{S}^{*}\right)=\tilde{A}(S)$.

Now, let $A$ be ar arbitrary almost $\mathcal{S}^{*}$-invariant set, i.e., $\mathscr{G}^{-1} A \sim A$ (equivalently, $g A \sim A$ ) for ail $g \in G^{\star}$. Consider the set

$$
B=\bigcap g 4
$$

where the intersection is taken over a $g \in \mathcal{S}^{*}$. Since $\mathcal{G}^{*}$ is a group, the $\operatorname{set} B$ must be $\mathcal{G}^{*}$-invariant. Again, since $\mathcal{G}^{*}$ ts countable, and each $g A$ is poquivalent to $A$, we hare $B \sim A$ We have thus established the following lemma.

Lemma 1. For any countable class $\mathcal{S}$ of transformatims [definition $2(f)]$, every almost S-invariant set is essentially $\mathcal{G}$-invart oni, i.e., $\widetilde{A(S)}=\widehat{A(S)}$.

Theorem 2, together with lexnma I and the observation that a sub-field that is yo-equivalent to a suffeient sub-field is itsel sufficient, leads to the following theorem.

Theorem 3. If $\mathcal{G}$ is a conntaile class of model-presoning transformations, then the sub-fielt $\mathcal{A}(\mathcal{S})$ of $\mathcal{S}$-invariant seis sufficient.

[Remark: Note that in Theorem 3 we have used the one to-oneness azd bimeasurability of our transformations.]

Before proceeding further, let us consider an example which shows that Theorem 3 is no longer lrue if we drop the conditiot. that $s$ is countable. 
Example 1. Let $y^{2}$ be a family of continuous distributions on the real line $Q$ where $\alpha$ is the $\sigma$-field of Borel-sets. Let $\mathcal{S}=\{g\}$ be the class of all one-to-one maps of $\mathscr{S}$ onto $\mathscr{C}$ such that If it is modei-preserviug. It is easy to check that the sub-field $\mathcal{A S})$ of $\mathcal{S}$-invariant sets consists of $\dot{\phi}$ and $\mathscr{C}$ only, and hence is not suficient. The sub-ficld $\mathscr{A}(\mathscr{G})$ of almost $\mathcal{G}$-invarjant sets to the same as $A$ and is suffoient.

Consider another example.

Exampie 2. Tuet $\mathscr{C}$ be the n-dimensional Euclidean space and 7 the class of all probability measures (on the $\sigma$-ieid $\mathscr{A}$ of all Borel-sets) that are symmetric (in the co-ordinates). If $s=\{g\}$ s the group of all permatations (of the co-ordinatos) then $\mathcal{A}(\mathcal{S})$ is the sub-field of all sets that are symmetric (in the co-ordinates) and is suficient. Since the empty sot is the oniy $p$-null set, the topo sub-fields $\mathcal{A}(S)$ and $\widetilde{A}(S)$ are the same here.

Given a probability model $(\mathscr{C}, A, x)$ we ask ourselves the following questions.

1 How wide is the group $S$ of all model-preserving transformations?

2. Is the sub-field $\widehat{A}(\mathcal{S})$ of almost $\mathcal{G}$-invariant setis suffeient?

3. If a least (minimal) sufficient sub-ficld $\mathcal{L}$ exists, then is it true that $\mathscr{L} \sim \widetilde{\mathcal{A}}(\mathcal{G})$ ?

4. What is $\widetilde{A}(\mathcal{S})$, when $\mathcal{S}$ is the olass of all transformations that partialiy preserve the model in a given manner?

\section{STATISTICAL MOTIVATION}

Suppose wo hare two different systems of measurement coardarites for the outcome of a statistical experiment, so that, if

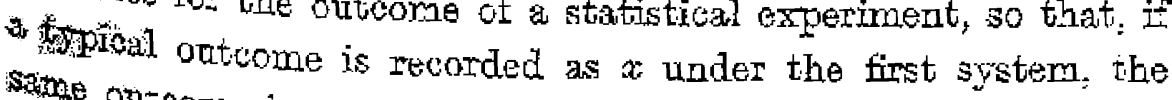
as oncoome is recorded as gx under the second system. Let us suppose forthur that the two statistical rariables $x$ and $g x$ 
have the same domain (sample space) $\mathscr{C}$, the same family $\mathcal{A}$ of events, and the same class of probability measure 20 . Furthermore, if $P \in \mathcal{O}$ holds for $x$, then the same probability measure $P$ also holds for $z x$. The second system of measurement co-ordinaties may then bo represented mathematically as a model-preserving transformation $g$ of the statistical model $(\mathscr{C}, A, 2 D$ ). The principle of invariance then stipulates that the decision rule should be invariant with respect to the iransformation g-and this irrespective of the actual decision problem. Tf the choice of a new sustem of measurement co-ordinates leaves the problem (whatever it is) entirely unaffected, then so also should be evory reasonable inference procedure.

Thus, if $g$ is model-preserving, the principle of iovariance leads to the invariance reduction of the model $(\mathcal{C}, \mathcal{A}, \mathcal{D})$ to the simpler model $(C, A, g)$, $\not)$, where $A(g)$ is the sub-field of $g$-invariant sets. To put it differently: the principle of invariance requires every decision function to be $g$-invariant or $\mathscr{A}(g)$-measureable. Consider now the olass $\mathcal{G}$ of all model-preserving iransformations $g$. Must we, following the prinoiple of invariane, insist that every decision rule be $S$-invariant (i.e. g-invariant for every geS) ? We have already noted in example I that, when 70 consists of a family of non-atomic measures, the class $\mathcal{G}$ is large enough to reduce the sub-field $A(S)$ of $\mathcal{S}$-inpariant sets to the trivial one (consising of only the empty set and the whole space). Obviously, we cannot (must not) reduce $A$ all the way down to $\mathcal{A}(\mathcal{S})$ or even to $\overline{A(s)}$-the sub-fiold of essentially $\mathcal{G}$-invariant sets. A logical compromise (with the principle) would be to reduce $A$ to the sub-field $\widetilde{A}(\mathcal{S})$ of all almost $\mathcal{S}$-invariant sets-that is to insist upon the decision function to bo almost $\mathcal{G}$-invariant.

The principle of sufficieney is another reduction principie of the ornibus type. If $s$ be a sufficient sub-field, then this prin. ciple tells us not to use a decision function that is not $S$-measur able. Suppose there exists a least (minimal) sufficient subField $\alpha$. Following the principle of sufficionoy, we reduce tho model $(\mathscr{C}, A, t)$ to the wodel $(\mathscr{R}, \mathcal{L}, \mathfrak{x})$. 
Which of the two reductions (invariance or sufficiency) is more extensive? In other words, what is the relation between $\widetilde{A}(\mathcal{S})$ and $\mathcal{L}$ ?

Theorem 1 tells us thain $\mathscr{R}(g)$ is aufficient (for each $g$ in $\mathscr{Q}$ ). Since 2 is the least sufficient sub-field we have (from the definition of $\infty$ )

$$
\bar{L} \subset \bar{A}(g) \text { for all } g \in Q
$$

Theorem 4 follows at once.

Theorem 4. $\quad \overline{\mathcal{L}} \subset \bigcap \overline{\mathcal{A}}(g)=\widetilde{x}(s)$.

[Remark: Theorem 4 does not establish the sufficiency of $\widetilde{A}(\mathcal{G}) . \quad$ [See example 1 in [3].]

We thus observe that the invariance reduction (in terms of the group $S$ of all model-preserving trarisformations) of a model can never bo more extensive than its maximal sufficiency reduction (if one such reduction is araidible). The prinoipal question raised in this paper is, "When is $\widehat{A(S)}$ equal to $\bar{x} ? "$ We shall show later tina in many familiar situations the sub-fields $\tilde{\mathcal{A}}(S)$ and $\alpha$ are essentially equal. Tris raises the question about the ngture of $\widetilde{A}(\mathcal{S})$, where $\underline{G}$ is the class of all trans Cormations that preserve the model partiolly in some whll-defined manner. This question is discussed in a later section. In the next two sections we give two alternative approuches to Theorem 4.

\section{WHEN A BOLNDEDLY COMPLETE SLFFICEEYT SUB-FIELD EXISTS}

Let us suppose that the suif-field $\mathcal{L}$ is sufficient and boundedly coxplete. We nced the following lemma.

Lemma 2. If $z$ is a bounded A-measurable funcion such that

$$
E(z ; P)=0 \text { for alt } P \in P \text {, }
$$

When, for alt bounded o-medwurable functions $f$, it is true that

$$
E(z f \mid P) \equiv 0 \text { for all } P \in \not p \text {. }
$$


The proos of this well-known result is omitted. Now, let $S$ be an arbitrarg nember of $\mathcal{E}$, and let $g$ be an arbitrary madelpreserving trensformation. Let $S_{n}=g^{-1} S$. Since $g$ is modelpreserving we have

$$
P(\delta) \equiv P g^{-1} S=P\left(S_{0}\right) \text { for all } P \varepsilon \% \text {. }
$$

Writing $I_{S}$ for the indicator of $S$, and noting that $I_{S}-I_{S_{0}}$ and $I_{S}$ satisfy the conditions for $z$ and $f$ in remma 2 , we at once have

$$
E\left[\left(I_{S}-I_{s_{0}}\right) I_{S} \mid P\right] \equiv 0 \text {, for all Pepo, }
$$

or

$$
P(S) \equiv P\left(S S_{0}\right), \text { for all } P \in \% \text {. }
$$

Henco,

$$
\begin{aligned}
& P\left(S \Delta S_{0}\right)=P(S) \div P\left(B_{0}\right)-2 P\left(S S_{0}\right) \\
& \equiv 2\left[P(S)-P\left(S S_{0}\right)\right] \\
& \equiv 0, \text { for all } P \in P \text {. }
\end{aligned}
$$

That is, for all $\delta e s$, the two sets $s$ and $g^{-1} g$ are $y 0$-equivalent. In other words,

$$
\alpha \overline{\mathcal{A}}(g)
$$

and sinee $g$ is an arbitrary element of $\mathcal{S}$ (the class of all modelpreserving transformations) we have the foliowing theorem.

Theorern 4 (a). If \& is a boundedly complate sufficient sth-jeld then $\alpha \subset \widetilde{A(S)}$.

[Remarle: Since bounded completeness of $\propto$ implies that $\alpha$ is the least sulicient sub-field, Theorem $4(a)$ is nothing but a special case of 'l'heorem 4 . The proof of Thesrem $4(a)$ is s'inple and amenable to a generalization to be discussed later.]

\section{THE DOMINATED CASE}

We make a slight digression to stato a useful lemma. Let $T$ be a measurable map of $(O C, M$ into $(\mathcal{L}: \mathcal{S})$. Let $P$ and $Q$ be two probability measures on $\mathcal{A}$ and let $P T^{-1}$ and $Q T^{-1}$ be the corresponding measuros on 2 . Suppose that $Q$ dominates $P$ and 
let $f=d P / d Q$ be the Radon-Nitodym dertvative defined on $\mathfrak{Q}$. It is thon clear thet $Q T^{-1}$ dominates $P T^{-1}$. Let $\tilde{h}=\left(\alpha P T^{-1}\right) \mid$ $\left(d Q T^{-1}\right)$. The function $h T$ on $Q 2$ (deffmed as $h T^{\prime}(x)=h(T x)$ ) is $T^{-1}(\mathcal{B})$-measurable and satisfics the following relation.

Lemma 3. $\quad h T=E\left(f \mid T^{-1}(g), Q\right)$,

i.e., $h T$ is the conditional ecpeclation of $f$, given $T^{-1}(B)$ and $Q$.

The prool of this well-known lemma consists of checking the identity

$$
\int_{E} f d Q \equiv \int_{B} h T d Q, \text { for al } B \in T^{-1}(A)
$$

Corollary. If $T^{-1}(Q)$ is $Q$-equolent to $\mathcal{A}$, then $f$ and $h T$ are Q-equivatent.

Now, returning to our problem, let $\mathcal{G}$ be the class of all transformations $g$ that preserves the model $(\mathcal{C}, \mathcal{A}, \not 2)$. Let us stppose that $y$ is dominated by some $\sigma$-finite measure. It foliows that there exists a countable collection $P_{1}, P_{2}, \ldots$, of elements in osuch that the convex combination

$$
Q=\Sigma_{c} P_{i}, \quad c_{i}>0, \quad \Sigma_{c i}=1
$$

dominates the family to. Lei $f_{F} \cdots d P / d Q$ be a fixed version of the Radon-Nikodym dexivative of $P$ will resped to $Q$. The factorization theorem for suffeicht statistics assorts thet a subfield $\mathcal{A}_{0}$ is sufficient if and only $i^{\prime} f_{P}$ is $\overline{A_{0}}$-measurable for every

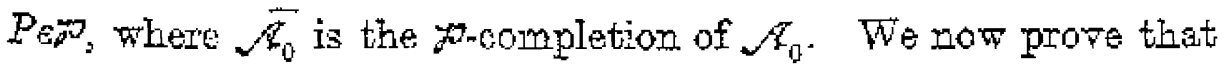
$f_{P}$ is $\widetilde{A}(G)$-measurable for every $P$. (The to-completion of $\widetilde{A}(G)$ is itself.)

Since $P g^{-1}-P$ for all $P Q P^{\prime}$, it follows that $Q g^{-1}=Q$, From Eemma 3 we have

$$
f_{P} g=D\left(f_{P}\left[g^{-1}(A), Q\right)\right.
$$

The assumption that $g$ is ono-to-one and bimeasurable implies that $g^{-1}(\mathscr{A})=\mathcal{A}$. Honce $f_{P} G=f_{P}$ a.e.w. [Q]. Since $Q$ domina-

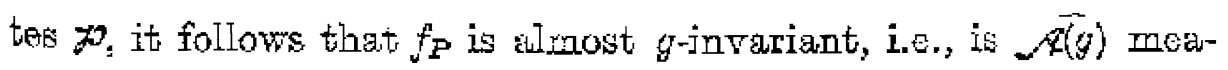


surible. Since, in the abote argument, $g$ is an arbitrary element of $\mathcal{S}_{3}$ wo have the following theorem. (Seo problem 19 on p. 258 in [6].)

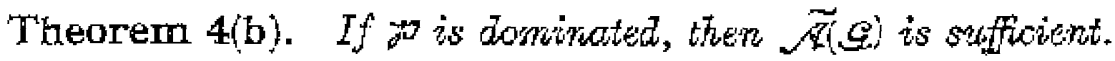

[Remain: Since, in the dominated set-rp, the least sufficient sub-field $\mathcal{L}$ always exists and since, in this set-rp, any sub-field containing $\mathcal{L}$ is necessarily sufficient, it is clear that Theorem 4(b) is nothing but an immediate corollary to Theorem 4. Also note that in the present proof (of Theorem $4(\mathrm{~b})$ ) we had to draw upon our supposition that $g$ is one-to-one and bimeasurable. This section has been written only with the object of drawing attention to some aspects of the problem.]

\section{EXAMPLES}

Example 3 : Let $y$ be a real random variable having a tniform distribution over the unit interval. For each $c$ in $[0,1)$ detine the iransformation $g_{c}$ as

$$
g c y=y+c(\bmod 1)
$$

In this example, 70 consists of $a$ single measure and each $g_{0}$ is model preserving (measure-preserving). If $s_{0}=\left\{g_{c} \mid 0<0<1\right\}$; then the only $S_{0}$-intariant sets are the empty set and the whole of the anit inte-val. Here, $\mathcal{A}\left(\mathcal{G}_{0}\right)$ is sufficient.

Remorl: In this case, there are a tery large number of measure-preserving urensformations that are not one-to-one maps of $[0,1]$ onto itself. For example, let $\alpha_{n}(y)$ be the $n$th digit in the decimal representation of $y$ and let

$$
g g=\sum_{k=1}^{\infty} \frac{a_{n_{k}}(y)}{10^{k}}
$$

winere $\{n\}$ is a fixed increasing sequence of natural numbers.]

Again, if $x$ has a fixed ontinuous distribution on the real line with oumulative distribution function $F$, then the class $\mathcal{S}_{0}$ of transformations $g_{c}$ defined as

$$
g_{c} x=F^{-1}[F(x)+0(\bmod 1)], \quad 0<0<1
$$


are all model-preserving for $x$. [In case $F$ is not a strictly increasing function of $x$, we define $F^{-1}(y)=\inf \left\{x \mid F^{2}(x)=y\right\}$.]

Thus, for any fixed continuous distribution on the real line, there always exists a large olass of measure-preserving transformations.

Example 4. Let $\mathscr{L}=[0, \infty)$ and let $\approx$ have 2 uniform distribution over the interval $[0,0)$, where $\theta$ is an unknown positive integer. Here 30 consists of a countable infinity of probability measures. For each $c$ in $[0,1)$, define the transformstion $g_{c}$ as

$$
g_{c} x=[x]-\{x-[x]+c(\bmod 1)\}
$$

where $[x]$ is the integer-part of $x$.

Here, edoh $g_{c}$ is model-preserving. The minimal (least) sufficient statistic $[x]$ is also the maximal-invariant with respect to the group $\mathcal{G}_{0}=\{g\}$ of model-preserving transformations defined above. Thus, if $\propto$ is the sub-field (least suffient) gencrated by $[x]$ and $\mathcal{A}\left(\mathcal{S}_{0}\right)$ is the stb-field of $\mathcal{S}_{0}$-invariant sets, we have

$$
\mathcal{L}=\mathscr{A}\left(\mathcal{S}_{0}\right)
$$

Now, if $\mathcal{G}$ is the class of all model-preserving transformations, then (as we have seen in Example 1) the sab-field $\mathcal{A}(G)$ will reduce to the level of triviality. (It will consist of only the empty set and the whole set Qs) Howeves, from Theorem 4 we have

$$
\bar{\pi} \subseteq \widetilde{A}(9)
$$

Since the group $\mathscr{S}_{0}$ has a decent structure, we can apply Stein's theorem (theorem 4 on p. 225 in [6]) to prove that

$$
A\left(\mathcal{G}_{0}\right) \sim \widetilde{A\left(\mathcal{S}_{0}\right)}
$$

Since $G_{0} \subset s$, we at once hare

$$
\widetilde{A}(\mathcal{G}) \subset \widetilde{\mathcal{A}\left(\mathcal{S}_{0}\right)}
$$


Putiting the relations (a), (c), and (d) together we have

$$
\widetilde{A}(\mathcal{S}) \subset \overline{\mathcal{L}}
$$

Putting (b) and (e) together we finally have

$$
\overline{\mathcal{E}}=\widetilde{A(G)}
$$

i.e., the least-sufficient sub-field (rather, the $x$-completion of any version of the least sufficient sub-field) and the sub-field of

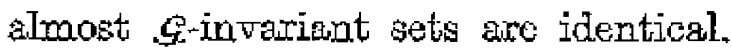

The chain of arguments, detailed as above, is of a general nature and will be used repeavedly in the sequel.

Example 5. Let $x$ be a normal variable with unit wariance and mean equal to either $\mu_{1}$ or $\mu_{2}$. Does there exist a non-trivial transformation of $x$ (the real line) into itself that preserves each of the two measures ? That the answer is "no" is seen as follows. Le' $\mathcal{G}$ be the class of all modcl-preserving transformations. In view of theorem $4, \widetilde{A}(S)$ contains the lesst auficient sub-field $\mathscr{C}$. But, in this example, the likelihood ratio (wibich is the least suffiecient statistic) is

$$
\exp \left[\left(\mu_{1}-\mu_{0}\right) x-\frac{1}{2}\left(\mu_{1}^{2}-\mu_{2}^{2}\right)\right]
$$

and this is a one-to-one measurable function of $x$. Thus, every set is almost $\mathcal{S}$-invariant. And this implies that every $g$ in $\mathcal{S}$ must be equivalent to the identity map.

Example 6. Lot $x_{1}, x_{2}, \ldots, x_{n}$ be $x_{\text {indepondent observations }}$ on a norma.l rariable with known mean and noknown stanaard deviation $\sigma$. Without loss of generality wo mat assume the mean to be zero. Let $\mathcal{G}_{0}$ be the grourp of all linear orthogonal transformations of the n-dimentional Euclidean space onto itzelf. Clearl $y$, every member of $S_{0}$ is model-preserving. That the class $\mathcal{G}$ of all model-preserving transformations is mach wider than $g_{0}$ is seen as follows. Let द:

$$
y_{i}=\phi_{i}\left(x_{i}\right): x_{i} \mid, i=1,2, \ldots, n_{2}
$$


where $\phi_{1}, \phi_{1}, \ldots, \phi_{r i}$ are arbitrary slew-symmetric (i.e., $\phi(x)$ $=-\phi(-x)$ for all $x$ ) functions on the real line that take only the two valnes -1 and +1 . It is easily checked that, whatever the value of $\sigma$, the two vectors $\left(x_{1}, x_{2}, \ldots, x_{n}\right)$ and $\left(y_{1}, y_{g}, \ldots, y_{n}\right)$ are identically distributed, i.e., the above transformation (though non-linear) is model-preserving. However, the sub-group $S_{0}$ is large enough to lead tos to $\Sigma 2$ - which is the least suffeient statistic-as the maximal invariant. In view of the decent structure of the sub-group $\mathcal{G}_{0}$, the arguments given for example 4 are again arailable to prove the equality of $\overline{\mathcal{E}}$ and $\widetilde{\mathcal{A}}(\mathcal{G})$.

\section{TRANSFORMATIONS OF A SET OF NORMAL VARIABLES}

This section is devoted to a study of the special case (model) of $n$ independent normal variables $x_{1}, z_{2}, \ldots, x_{n}$ with equal unknown means $h$ and equal unknown standard deviations $\sigma$. Fenee $\Sigma x_{i}$ and $\Sigma x_{i}^{2}$ jointly constitute the least sufficient statistic.

$L_{F} \vec{x}$ is the 7 -completion of the sub-field $\alpha$ indueed by $\left(\Sigma x_{i}\right.$, Exis), then we krow from Theorem 4, that

$$
\overline{\mathcal{L}} \subset \widetilde{\mathcal{A}}(\mathcal{S})
$$

Where $S$ is the class of all the model-preserving transformations of $\boldsymbol{x}=\left(x_{1}, x_{2}, \ldots, x_{n}\right)$ to $\boldsymbol{y}=\left(y_{1}, y_{2}, \ldots, y_{n}\right)$. For any model-preserving transformation from $x$ to $y$, we, therefore, have

and

$$
\Sigma x_{i} \sim \Sigma y_{i}
$$

$$
\Sigma x_{*}^{2} \sim \Sigma y_{\tilde{*}}^{2}
$$

i.e., the statistic $\left(\Sigma \alpha_{i}, \Sigma c_{i}^{2}\right)$ is almost $\mathcal{E}$-invariant. If we can demonstrate the existence of a 'decent' ant-group $\mathcal{G}_{0}$ of $\mathcal{S}$ for which the statistic $\left(\Sigma x_{i}, \Sigma x_{i}^{*}\right)$ is the maximal invariant, then (following the method of proof indicated in examples 1: and 6) We can show that $\widetilde{\mathcal{L}}$ is indeed equal to $\widetilde{\mathcal{A}} \mathcal{G})$.

Let $\mathcal{S}_{0}$ be the sub-group of all linear model-preserving trarsformations. Do there exist nor-trivial linear model-preserving 
transformations (i.e., linear transformations that are not a permutation of the co-ordinates) ? That the answez is " $y e s "$ is seen as follows. Iet $\mathcal{K}=\{M\}$ be the family of ail orlhogonal $n \times n$ matrices with the joitial row as

$$
\left(-\frac{1}{\sqrt{n}}, \frac{1}{\sqrt{n}}, \ldots, \frac{\mathrm{I}}{\sqrt{n}}\right)
$$

Now, if $x=\left(x_{1}, x_{2}, \ldots, x_{n}\right)$ are independent $\left.N(\mu, \sigma)\right)_{s}$ and we define

$$
\boldsymbol{y}^{\prime}=\boldsymbol{M} \boldsymbol{d \boldsymbol { x } ^ { \prime }}
$$

(where $x^{\prime}$ is the corresponding colnmon vector), then the $y_{i}{ }^{\prime}$ are independent normal variakles with equal standard deviation $\sigma$ and with means as follows:

$$
E\left(y_{1}\right)=\sqrt{n} \mu, \quad E\left(y_{i}\right)=0, \quad i=2,3, \ldots, n .
$$

Thus, for an arbitrary pair of members $M_{1}$ and $M_{2}$ in $\mathscr{H}_{3}$ we note that $\boldsymbol{M}_{1} \boldsymbol{x}^{\prime}$ and $\boldsymbol{M}_{\mathrm{g}} \boldsymbol{x}^{\prime}$ are identically distributed (whatever the: valtues of $\mu$ and $\sigma$ ). Therefore, the two vectors $x^{\prime \prime}$ and $M_{2}^{-1} M_{1} x$ are identically distributed for each $\mu$ and $\sigma$. In other words the linear transformation defined by the matrix

$$
M M_{2}^{-1} M_{1} \quad\left(=M_{0} M_{1}\right.
$$

since $M_{2}$ is orthogonal) is model-preserving for exch pair $M_{1}, M_{2}$ of members from $N$.

[Rematk : Later on we shall have some use for this way of generating members of $\varepsilon_{0}$.]

For example, the $4 \times 1$ matrix

$$
\left(\begin{array}{rrrr}
1 / 2 & 1 / 2 & 1 / 2 & -1 / 2 \\
1 / 2 & 1 / 2 & -1 / 2 & 1 / 2 \\
1 / 2 & -1 / 2 & 1 / 2 & 1 / 2 \\
-1 / 2 & 1 / 2 & 1 / 2 & 1 / 2
\end{array}\right)
$$

defines a member of $s_{0}$ for $n=4$. 
Let $\not$ be a typical $n \times n$ matrix that defines a model-preserving Iined transformation. We are going to make a brief digression about the nataro of $I I$. From the requirement that each co-ordinate of

$$
y^{\prime}=B x^{\prime}
$$

has mean $\mu$ and atandard deviation $\sigma$, we an once have that the elements in each row of $B$ must add up to unity and that their squares also must add ng to wity. From the mutral indepondence of the $3{ }^{3} \mathrm{~s}$, it follows that the row vectors of $A$ must be mutually orthogonal. Thus; $H$ must be an orthogonal matrix with wit row stms. Now, for each wodel-preserving $E$, its interse

$$
B^{-1}\left(=H^{\prime}, \text { sinee } H\right. \text { is orthogonal) }
$$

is-necessarily also model-preserving and, thus, the columes of A must also add up to unity, eto.

It is easily checked that the sub-group $\mathcal{S}_{0}=\{Z\}$ of linear model-preserving transformations of the $n$-space onto itself Inay also be characterized as the class of all linear transformawons that preserve both the sum and the sum of squares of the co-ordinates. The anthor came to leatn that $G$. W. Hagsastrom of the Univarsity of Chioage had come apon these matrices from this point of riew and had a brief discussion on them in an umpublished worl of his. Wo call sucin matrices by the name Hagostroni-matrix]

Going back to our problem, we hare to demonstrate that the statistic $T=\left(\sum x_{1}, \Sigma x_{i}^{2}\right)$ is a mixinal invariant with respect to the sab-group $\varsigma_{0}=\left\{H_{j}\right.$ of model-preserving linear transformations. For this we have only to prove that if $a=\left(a_{1}, a_{m}, \ldots, a_{n}\right)$ and $b=\left\langle b_{1}, b_{s}, \ldots, b_{n}\right\rangle$ are any two points in $n$-space such that $T(a)=T(b)$ then there exists a Haggstrom-matrix $H$ suoh that

$$
b^{\prime}==B a^{\prime} \text {. }
$$

Let $M_{I}$ and $M_{2}$ be two arbitrary members of $\mathscr{N}$-the class of aII ortiogonai $n \times n$ matrices with the leading row as $(1 / \sqrt{n}, \ldots$, 
I $(\sqrt{n})$. If $\alpha=M_{2} a^{\prime}$ and $\beta=M_{2} b^{\prime}$, then we have, from $T(\alpha)$ $=T(b)$, that $\alpha_{1}=\beta_{1}$ that and that

$$
\sum_{2}^{m} \alpha_{i}^{2}=\frac{n}{2} p_{i}^{2}
$$

It follows that there exists an $(n-1) \times(n-1)$ ortinogonal matrix theit will transform $\left(\alpha_{2}, \alpha_{3}, \ldots, \alpha_{n}\right)$ to $\left(\beta_{5}, \beta_{2}, \ldots, \beta_{n}\right)$. And this, in turn, impies that there exists an $n \times n$ orthogonal matrix $K$, with the first row as $(1,0,0, \ldots, 0)$, such that

$$
\beta^{\prime}=K \alpha^{\prime} \text {. }
$$

Thus, the transformation $M_{2}^{-1} K M_{1}$ takes $a$ into $b$. Now, note that, since $Z$ is an orthogonal matrix with the initial row as $(1,0,0, \ldots, 0)$, the matrix $K M_{\mathrm{t}}$ is orthogonal with its initial row as $(1 / \sqrt{n}, 1 / \sqrt{n}, \ldots, 1 / \sqrt{n})$, j.e. $E M_{1} \in \mathcal{M}$. In other words, there exists a matrix of the form $M_{2}^{-1} M^{2}$ (with $M_{2}$ and $M$ belonging to M) which transforms $a$ into $b$. We have alrewdy noted that all such matrices bolong to $\mathcal{G}_{0}$, and this complotes our proof that $\left(\Sigma x_{\dot{i}}, \Sigma x_{i}^{0}\right)$ is a marimal invariant with respect to $\mathcal{G}_{0}$.

In example 6 of the previous section, we considered the particular case of the foregoing problem where $\beta$ is known. Consider now the other partieular case where $\sigma$ is lnown and $\mu$ is the only unkown parameter. In this osse $\Sigma s$ is the least scfficient statistic. Now, it is no longer possible to produce 2 stb-group $\mathfrak{S}_{0}$ of linear modol-preserving transformations such that $\Sigma s_{i}$ is the raximal jnvarinant with respect to $S_{0}$. This is because every linear model-preserving transformation rast necessarily be orthogonal and would thus preserre $\Sigma x^{2}$ also. We proceed as follows.

Srapose (withou loss of generality) that $\sigma=I$. Let $y^{\prime}$ $=M x^{\prime}$ where $M$ is a lixed member of the class $M$ of orthogonal $n \times n$ matrices with the initiel row as $(I \sqrt{n}, \ldots, 1 / \sqrt{n})$. Observe that the $y_{1}$ 's are mutually independent and that $y_{2}, y_{3}, \ldots, y_{k}$ are standard normal variables. Iet $I$ stand for the cumulative distribution function of a standard normal variable. Let. 
$c_{3}, \ldots, c_{n}$ be arbiurary constants in $[0,1)$. If we dofine $z_{1}=y_{1}$ and

$$
w_{1}=F^{-1}\left[F\left(y_{j}\right)+c_{\mathrm{i}}(\bmod 1)\right], \quad \dot{m}=2,3, \ldots, n,
$$

then $z=\left[z_{1}, z_{2}, \ldots, z_{n}\right]$ has the same distribution as that of $y$ and so it follows that $z^{\prime}$ and $M^{-1} z^{\prime}$ are identically distributed. Observe thaì we have described above a model-preserving transformation corresponding to each $(n-I)$-vector $\left(c_{3}, c_{3}, \ldots, c_{n}\right)$ with the $c_{i}{ }^{\prime} \mathrm{s}$ in $[0,1)$. It is easly checked that $\sum x_{i}$ is a maximal invariant with respect to the above class of model-preserving transformations.

\section{PARAMETER-PRESERVING TRANSFORMATYONS}

Let $\gamma=\gamma(P)$ be the parameter of interest. That is, the experimenter is jnterested only in the characteristic $\gamma(P)$ of the mossure $P$ (that actually holds) and considers all o ther cetaits abort $P$ to be irrelevent (nuisance parametexs). Wo define a $\gamma$-preserving transformation as follows.

Definition. The trensformation [see Definition 2(f)] $g$ is $\gamma$-preserving if $\gamma\left(P g^{-1}\right)=\gamma(P)$, for wll $P \in \not \partial$.

Lf $g$ is $\gamma$-preserving then so also is $g^{-1}$. The composition of any two $\gamma$-preserving transformations is also $\gamma$-preserving. Lat $\mathfrak{A}_{\gamma}$ bo the group of all $\gamma$ preserving transformations.

In the particular case where $\gamma(P)=P$, the $\gamma$-preserving transformations are what wo have so far been calling modelpreserving. If $\mathcal{G}$ is the class of all model-preserving transformations, then note that $\mathscr{A} \subset \mathcal{B}_{\gamma}$ for every $\gamma$.

If $\gamma$ is the parame ance leads ta the reduction of $\mathscr{A}$ to the sab-field $\widetilde{A}\left(\mathcal{G}_{y}\right)$. This section is devoted to a study of the sub-field $\tilde{\mathcal{A}}\left(\mathcal{G}_{\gamma}\right)$.

Sinco $\mathscr{G} \subset \mathcal{G}_{r y}$ we have

$$
\widetilde{A}\left(\mathcal{B}_{\gamma}\right) \subset \widetilde{\mathcal{X}}(\mathcal{S})
$$

Let as suppose that the least ouffejent sub-hidd $\mathcal{L}$ exists. Wo have discussed a number of examples where $\widetilde{A}(G)$ and $\mathscr{L}$ are 
identioal. TThe arthor believes that the above identification is true under very general conditions.] In ail such situations we therefore have

$$
\widetilde{A}\left(\mathfrak{s}_{\gamma}\right) \subset \widetilde{\infty}
$$

That is, when the interest of the experimenter is concentrated on some parbicnlar characteristio $\gamma(P)$ or $P$, the principle of invariano will usually reduce the data more extensively than the principle of sufficiency.

Theorems $4,4(a)$ and $4(b)$ tell as that

$$
\bar{L} \subset \widetilde{A}(\mathcal{G})
$$

The following thoorem gives us a similar lower bound for $\widetilde{R}(\mathcal{G})$.

A set $A$ is called $\gamma$-oriented if for crery pair $P_{1}, P_{y} \in P^{\prime}$ such that $\gamma\left(P_{1}\right)=\gamma\left(P_{2}\right)$, it is twue that $P_{1}(A)=P_{2}(A)$. In oliher worcs, a $\gamma$-oriented set is one whose probability depends on $P$ through $\gamma(P)$. A sub-field is $\gamma$-oriented if every member of the sub-field is. The following theorem* is a direct generalization of Theorem $\mathbf{A}(\mathrm{a})$.

Theorem 5. Lrt $\mathcal{G}_{\gamma}$ be the class of all $\gamma$-preserving transforma tions and lat $A$ be a aub-field that is

i) $\gamma$ roriented and

ii) contained in the boundedty complete sufficient sub-fidd $\mathscr{L}$ (which exists).

Then,

$$
\left.a \subset \widetilde{\mathscr{A}} \mathcal{G}_{2}\right)
$$

Proof: Let $g \varepsilon_{\gamma}$ and $B e_{\not Z}$ and let $E_{0}=g^{-2} \not B$

Then

$$
\begin{aligned}
P\left(B_{0}\right) & =P\left(g^{-1} B\right) \quad\left(\because \quad B_{0}=g^{-1} B\right) \\
& =P g^{-1}(B) .
\end{aligned}
$$

* The arthor wizhos to thank Professor W. J. Hall of the Criversity of Xort. Carolina for certain comments that eventuedly led to thie thoorem. 
Since $g$ is $\gamma$-preserving, we have $\gamma\left(P g^{-1}\right)=\gamma(P)$. And since $B$ is $\gamma$-oriented wo have

Therefore,

$$
P g^{-1}(B)=P(B) \text {. }
$$

$$
P\left(B_{0}\right)=P(E) \quad \text { or } \quad R\left(I_{B}-I_{E_{0}} \mid P\right)=0 .
$$

The rest of the proof is the same as in Theoreso $4(a)$.

In the next section we repertedly rse the above theorem.

\section{SOME TYPICAL INYARIANCE REDUCTIONS}

Let $x_{1}, x_{2}, \ldots, x_{n}$ be $x$ independent and identioal normal rariables with unknown $\mu$ and $\sigma$. Suppose the parameter of interest is $\sigma$. Lot $\mathcal{G}_{\sigma}$ bo the class of all $\sigma$-preserving transformathons. If $z$ is the mean and $s$, the standard deviation of the $n$ observations, then the pair $(z, s)$ is a complete sufficient statistic. Also $s$ is $\sigma$-oriented. From Theorem 5 , we then bave that $s$ is almost $\mathcal{G}_{\sigma}$-intariant. Thas, the principle of invariance cannot reduce the data beyond $s$. That $s$ is indeed the exact (upto equivalence) attainable limit of invariance reduction is shown as follows.

Lot $\{H\}$ be the class of all $x_{x} \times n$ Haggstrom matrices (see section s), i.e., each $H$ is an orthogonal rastrix with unit row and column strms. Let $a$ be an arbitrary rea number and let $i$ stand for the $n$-vector $(1,1, \ldots, I)$. Consider all linear transFormations of the type

$$
y^{\prime}=B x^{\prime}+(c i)^{\prime}
$$

If $\mathcal{G}_{\sigma}^{*}$ is this class of transformations, then is is easily verified that $S_{0}^{*}$ is a sub-group of $\mathcal{S}_{-}$. Thet $\Sigma\left(x_{i}-x\right)^{2}$ is a maximal intariant with respect to the sub-group $\mathfrak{S}_{\theta}^{*}$ of $\sigma$-preserving transformations is seen as follows.

Lat $a=\left(a_{1}, a_{2}, \ldots, a_{n}\right)$ and $\boldsymbol{b}=\left(b_{1}, \tilde{b}_{2}, \ldots, b_{n}\right)$ be two arbitrary Nectors scin that

$$
\Sigma\left(\alpha_{i}-\bar{a}\right)^{2}=\Sigma\left(\bar{b}_{i}-\bar{b}\right)^{2}
$$

Iftc $=\bar{b}-\bar{a}$. Then the two reetors $a+c i$ and $b$ hare equal sums ard sums of squares (of co-ordinates). Hence there exists a 
Faggstrom matrix $B$ that transforms $a$ - $+c i$ into $b$. In orter words, the transformation

maps $a$ into $b$.

$$
y^{\prime}=B x^{\prime}-(c a)^{\prime}
$$

If $\mathscr{B}(s)$ is the sub-field goncrated by $s$, then we have just

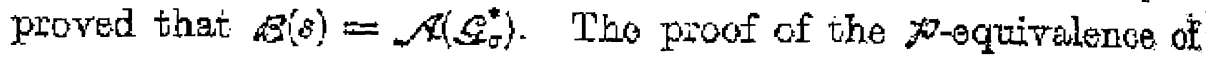

$$
B(s) \text { and } \widetilde{A}\left(\mathcal{S}_{6}\right)
$$

will follow the familiar pattern set up earlier for example 4 in section 7 .

Now, suppose that our parameter of interest is $\gamma=\mu j p$ The statistic $z / s$ is $\gamma$-oriented and hence (Theorem 5) is almost $\mathcal{S}_{\gamma}$-invariant, where $\mathcal{S}_{\gamma}$ is the group of all $\gamma$-preserving transformations.

Consider now the sub-gronp $\mathcal{G}_{\gamma}^{*}$ of all linear transformations of the type

$$
y^{\prime}=c H x^{\prime}
$$

where $c>0$ and $H$ is a Haggstrom matrix.

It is easily chocked that the maximal invariant with respect to the sub-group $S_{\gamma}^{*}$ is the statistio $\vec{x} / s$ and hence, the sub-fidd

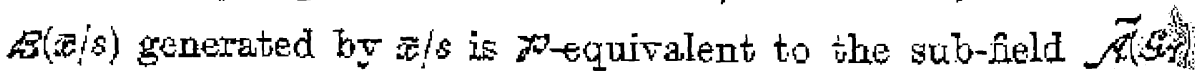
of all almost $\mathcal{G}_{\gamma}$-invariant sets.

The case where our parameter of interest is $|\mu| i \sigma$ is similar. Once again we observe that the statistic $\mid z J / s$ is oriented towards ; $\mu / \sigma$. Hence, malking use of Thoorem 5 and observing that $[x[/ s$ is the maximal invariant with respeot to the subgroup of linear parameter-preserping transformations of the form

$$
y^{\prime}=c B x^{\prime}(c \neq 0, B \text { a Haggstrom matrix }) .
$$

we are able to show that the inveriance reduction of the datil is to the statistio $|x| / s$. 


\section{SOME EINAL REMARKS}

a) In statigtioal literature, the principle of invariance has been rused in a rather half-hearted manner (see, for example, [5] and [B]). We do not find any consideration given to the present project of reducing the data with the halp of the whole class $G_{x}$ of $\gamma$-preserving transformations. In fact, the question of how extensive the class $s_{\gamma}$ can be has esoaped general attention. One uscally works in the framework of a relatively small and simple sub-group of $\mathcal{G}_{\gamma}$ and invokes simultaneousily the two different principles of suffiency and invariance for the purpose of ariving at a satisfactory data reduction. The main objoct of the prosent article is to inrestigate how far the principle of invariance by itself can taife as.

b) The min limitation of the principle of suminency is that Th does not recognize nuisance paramoters. Severai attemptz have been made to generalize the idea of sufficiency so that one gets an effective data reduction in the presence of nuisance parameters. Not mich success has, howcter, been achieved in this direction.

c) On the other hand, the invariance principle uscally falls to preces when faced with a discrete model. The Bemonlli expenmental set-up is one of the rame disorete models that the unvariance prine; ple can tackle. If $x_{1}, x_{2}, \ldots, x_{n}$ are $n$ independent zero-one variables with probsbilities $\theta$ and $1-9$, then all permutations (of co-ordinates) are model-preserving. And they reduce the data directly to the least sufficient statistic $r=x_{1}+\ldots+-x_{n}$. take however, the following simple example. Iet $x$ and $y$ be independent zero-one variables, where

and

$$
P(x=0)=0, \quad 0<\theta<I_{*}
$$

$$
P(y=0)=1 / 3 \text {. }
$$

How the identity-map is the only available model-preserving transformation. The principle of suffiency reduces the data impodiately to $x$. 
d) The object of this article was not to make a critical evaluation of the twin principles of data reduction. Yet, the author finds it hard to refrain from observing that both the principles of sufficiency and invariance are extremely sensitive to changes in the model. For example, the spectacular data reductions we have achieved in the many examples considered here become totally unavailable if the basic normality assumption is changed ever so slightly.

\section{References}

[I] Bahadur, R. R. "Suffieiency and Statistical Decision Functions," Ann. Math. Statist., 25 (1954), 423-462.

[2] Basu, D. "Sufficiency and Model-Preserving Transformations," Inst. of Statistics, Mimeo Series No. 420, Univ. of North Carolina, Chapel Hill, 1965.

[3] Burkholder, D. L. "Sufficiency in the Undominated Case," Ann. Math. Statist., 32 (1961), 1191-1200.

[4] Farrell, R. H. "Representation of Invariant Measures," Illinois J. of Math., 6 (1962), 447-467.

[5] Fall, W. J., R. A. Wijsman, and J. K. Gosh. "The Relationship between Sufficiency and Invariance," Ann. Math. Statist., 86 (1965), 575-614.

[6] Lehmann, E. L. Testing Statistical Eypotheses, John Wiley and Sons, Now York, 1959.

(Received Sept. 17, 1965.) 